\title{
Relay Cross Metathesis for the Iterative Ascent of the Terpenoids
}

\author{
Karim A. Bahou, ${ }^{[a]}$ D. Christopher Braddock, ${ }^{[a]}$ Adam G. Meyer, ${ }^{[b]}$ G. Paul Savage ${ }^{[b]}$ and Zhensheng \\ Shi[a]
}

\begin{abstract}
We report the design and implementation of a relay cross metathesis (ReXM) reaction for the ascent of the terpenoids in an iterative protocol. The method features the reaction of naturally occurring terpenoid building blocks - with preexisting trisubstituted olefins - which combine to construct a new trisubstituted olefin resulting in a five carbon unit homologation. Subsequent functional group manipulation allows for the method to be repeated in an iterative fashion. The method is used for the synthesis of a diterpene-benzoate macrolide of biogenetic relevance to the bromophycolide family of natural products.
\end{abstract}

\section{Introduction}

Terpenoids, consisting of 'head-to-tail' and 'head-to-head' arrangements of five-carbon isoprene units, are a diverse and very large class of linear and (poly)cyclic naturally-occurring biomolecules with more than 40,000 distinct chemical structures, thereby accounting for approximately $60 \%$ of known natural products. ${ }^{[1]}$ They mediate vital biological functions including light harvesting and photo-oxidative protection, lipid membrane modulation, electron transport, intercellular signalling as hormones, and interspecies defence amongst others. ${ }^{[2]}$ Traditional herbal remedies from plants have utilized the medicinal benefits of terpenoids for centuries, ${ }^{[1]}$ with the subsequent development of terpenoid derivatives (e.g., steroidal medicines) as blockbuster drugs in the 20th century through to the present day. ${ }^{[3]}$ While a comprehensive account of their biogenesis is beyond the scope of this document, it is important to note that (poly)cyclic terpenoids all arise from their linear precursors. ${ }^{[4]}$ Nature assembles these linear precursors by sequential addition of $\mathrm{C}_{5}$ units of isopentenyl pyrophosphate (IPP) to $\left(\mathrm{C}_{5}\right)_{n}$-terpenyl pyrophosphates in the mevalonate pathway. ${ }^{[5]}$ These linear terpenoids are important in their own right, where polyprenols ${ }^{[6]}$ are constituents of nearly every living cell, and the related dolichols ${ }^{[7]}$ play vital roles in peptide glycosidation. ${ }^{[8]}$ However, despite the long-term recognition that these linear compounds are essentially $\mathrm{C}_{5}$-repeating isoprene units, a general and iterative chemical protocol for their synthesis - using naturally-occurring, terpenoid building blocks - does not exist. ${ }^{[9]}$ Herein, we report the design and execution of an olefin metathesis reaction to achieve the above aim.

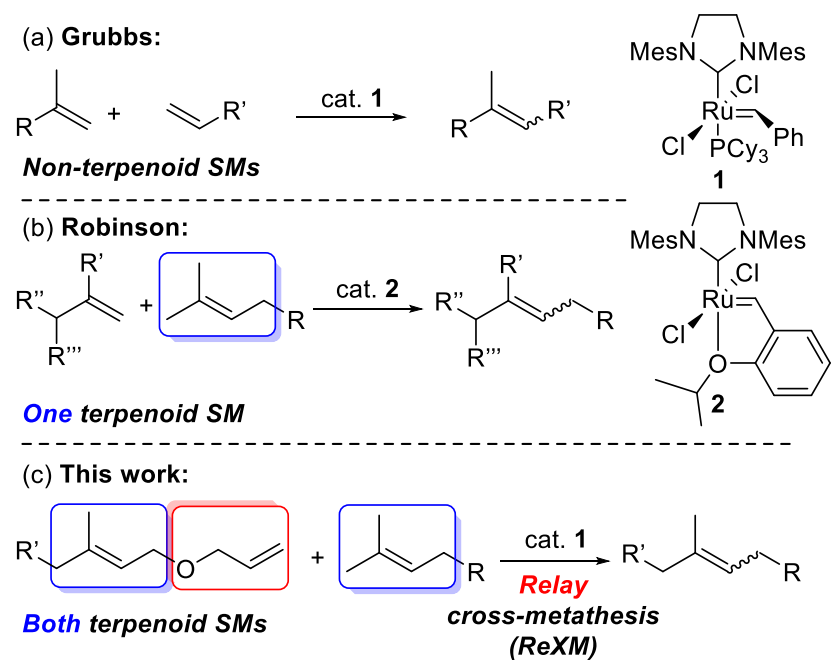

Figure 1. Ruthenium benzylidene-catalysed cross metathesis reactions to produce unfunctionalized trisubstituted olefins.

[a] Dr. K. A. Bahou, Prof. Dr. D. C. Braddock, Z. Shi

Department of Chemistry, Molecular Science Research Hub

White City Campus, 80 Wood Lane, London W12 0BZ, UK

E-mail: c.braddock@imperial.ac.uk

[b] Dr. A. G. Meyer, Dr. G. P. Savage

CSIRO Manufacturing, Jerry Price Laboratory, Research Way,

Clayton, Victoria, 3168, Australia 
The introduction of well-defined ruthenium benzylidene pre-catalysts for olefin metathesis has had a major impact on organic synthesis. ${ }^{[10]}$ Such catalysts are widely used to accomplish the ring-closing metathesis of disubstituted, trisubstituted and even tetrasubstituted olefins. ${ }^{[11]}$ In contrast, and quite surprisingly, there are only three reports on the formation of unfunctionalized trisubstituted olefins by cross metathesis using ruthenium benzylidene pre-catalysts. ${ }^{[12]}$ Grubbs and co-workers initially showed that ruthenium pre-catalyst 1 was competent for the cross metathesis of (non-terpenoid) geminally disubstituted olefins with terminal olefins (Figure 1a). ${ }^{[13-14]}$ Subsequently, Robinson and co-workers showed that the cross metathesis of sterically challenging allyl branched 1,1disubstituted olefins performed considerably better using a (terpenoid) prenyl rather than an allyl partner using pre-catalyst 2 (Figure 1b). ${ }^{[15]}$ Herein, we report an unprecedented olefin-olefin combination to form unfunctionalized trisubstituted olefins by cross metathesis (Figure 1c). The method utilizes two different trisubstituted olefins, one of which is a trisubstituted allylic alcohol furnished with a relay ${ }^{[16}$ - allowing the synthetic convenience of using readily available and inexpensive terpenes such as geraniol as building blocks - while the non-relay partner is an unfunctionalized trisubstituted olefin. ${ }^{[17]}$ This relay cross metathesis reaction (which we have dubbed "ReXM") distinguishes itself from the very limited literature precedent for such reactions by being the first such example to form an isolated, unconjugated, alkene where all previous reports have formed conjugated alkenes. ${ }^{[18-19]}$ Moreover, by judicious selection of the non-relay partner - in this case as another readily available terpene building block (vide infra) - an iterative ascent of the terpenoids from monoterpene to sesquiterpene to diterpene is demonstrated.

\section{Results and Discussion}

To ascend the terpenoids, we envisioned the ReXM of a suitably derivatized $O$-allyl geraniol (or nerol) to a naturally occurring monoterpene. Accordingly, $O$-allyl geraniol-derived ${ }^{[20]}$ epoxide $3 \mathbf{a}$, diol $\mathbf{4}$ and acetonide $5 \mathbf{a}$, and also $\mathbf{O}$-allyl nerol-derived epoxide $\mathbf{3 b}$ were prepared as potential relay partners (Figure 2). Des-allyl alcohol $\mathbf{5 b}$ and 'truncated' alkene $\mathbf{5 c}$ were also prepared as control substrates. For preliminary studies, we elected to use readily available prenyl acetate (6) as a proof-of-principle cross metathesis partner. It is well established that trisubstituted olefins - classified as Type III olefins ${ }^{[21]}$ - do not homodimerize, and this prompted us to use such olefins in excess with the expectation that this would facilitate the desired cross metathesis. Much to our delight, epoxide 3a underwent smooth ReXM using 10 mol\% 1 with neat prenyl acetate (5 equiv.) at $50{ }^{\circ} \mathrm{C}$ to provide functionalized geranyl acetate 15 (Table 1, entry 1). A comparison of the use of trisubstituted olefin 7 versus terminal olefin 8 under the same conditions (entries 2-3) with relay epoxide $\mathbf{3 a}$ demonstrated that the use of a trisubstituted olefin as the cross metathesis partner to produce the desired product 16 is beneficial under these conditions, in line with the observations of Robinson and co-workers. ${ }^{[15]}$ Whereas, $\beta, \beta$-dimethyl styrene 9 and prenyl benzene 10 were unreactive under the reaction conditions - leading only to truncated product 17 (entries 4-5) - homoprenyl benzene 11 gave ReXM product 18 in high yield (entry 6). Sensitivity to $\alpha$-branching was also observed using substrate 12 (entry 7 ). Readily available prenylacetone 13 gave the ReXM product 19 (entry 8). Diol 4 unexpectedly failed to undergo ReXM (entry 9), resulting in truncated compound 20a and isomerized product 20b (implicating catalyst decomposition to a ruthenium hydride species). ${ }^{[22]}$ Acetonide 5a however, participated cleanly in ReXM reactions (entries 10-11) to provide the desired products 21 and 22 without complication. Control experiments with acetonides $\mathbf{5 b}$ and $\mathbf{5 c}$ (entries 12-13) verifies the vital role of the relay in this ReXM process, and a comparison of the reactions of $E$-epoxide $\mathbf{3 a}$ and Z-epoxide $\mathbf{3 b}$ (entries 14-15) establish the olefin geometry in the relay partner as unimportant. In all successful ReXM cases the products were obtained with moderate (ca. 2-3:1) and comparable E-olefin selectivity ${ }^{[23]}$ to trisubstituted olefins previously prepared by cross metathesis with ruthenium benzylidene pre-catalysts (c.f. Figure 1 a,b). ${ }^{[13-15]}$

A possible catalytic cycle for the ReXM process (Scheme 1) using representative epoxide $\mathbf{3 a}$, invokes Diver ${ }^{[18]}$ for the conversion of $\mathbf{A}$ to $\mathbf{B}$ with loss of dihydrofuran $\mathbf{2 4}$. The regioselective reactions of ruthenium species of type $\mathbf{B}$ with trisubstituted olefins have been proposed by Robinson, ${ }^{[15]}$ which would produce ReXM products, and ruthenium isopropylidene $\mathbf{C}$. In this scenario, the catalytic cycle would be closed by re-initiation of ruthenium isopropylidene $\mathbf{C}^{[14]}$ on the terminal olefin of relay partner $3 \mathbf{a}$ with concomitant loss of isobutylene $25^{[24]}$

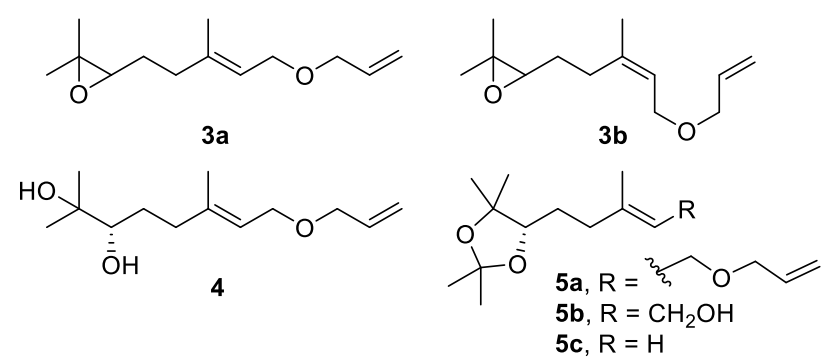

Figure 2. Structures of epoxides $\mathbf{3 a - b}$, diol $\mathbf{4}$ and acetonides $\mathbf{5 a - c .}$

Having demonstrated successful ReXM between derivatized $O$-allyl geraniols and nerols with trisubstituted olefins, we selected citral (26) - a monoterpene with two electronically distinguished olefins - as a readily available and inexpensive monoterpene cross metathesis partner ${ }^{[25-26]}$ to allow for the ascent of the terpenoids.

Starting with the previously identified conditions, initial attempts at ReXM between relay ( $S$ )-3a and citral (26) were unsuccessful (Table 2 , entry 1). Increasing to 10 equivalents of 26 (entry 2), at reduced temperature (entry 3 ) or reduced catalyst loading (entry 4 ) also failed. In these attempts, truncated olefin 17 was observed by ${ }^{1} \mathrm{H}$ NMR of the crude reaction material, along with characteristic signals 
corresponding to 2,3-dihydrofuran ${ }^{[27]}$ as the likely ruthenium hydride-induced isomerization of by-product $\mathbf{2 4}$, implicating catalyst decomposition.

Known hydride scavengers 1,4-benzoquinone (pBQ, entry 5) and $\mathrm{AcOH}$ (entry 6) were therefore explored as possible additives for the reaction. ${ }^{[28]}$ Pleasingly, the use of $\mathrm{AcOH}$ was beneficial, and $\mathrm{C}_{10}$-monoterpene epoxide (S)-3a and $\mathrm{C}_{10}$-monoterpene acetonide 5a now underwent smooth ReXM with $C_{10}$-monoterpene citral (26) to provide $C_{15}$-sesquiterpenes 27 and 28 in good yields (entries 6-7).

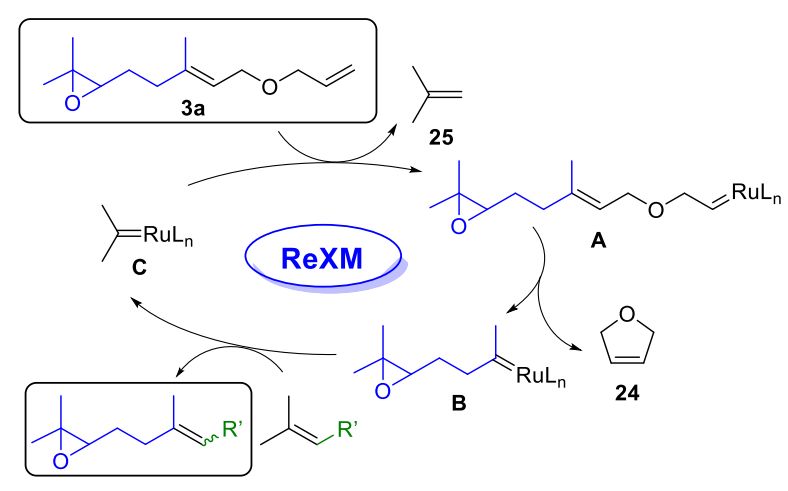

Scheme 1. Possible catalytic cycle for the ReXM reaction. 
Table 1. Reaction scope for the ReXM of $O$-allyl geraniol-derived epoxide $\mathbf{3 a}$, diol $\mathbf{4}$ and acetonide $\mathbf{5 a}$, and $\mathbf{O}$-allyl nerol-derived epoxide $\mathbf{3 b}$ as potential relay partners with trisubstituted olefins 6-14 using GII (1). ${ }^{[a]}$

\begin{tabular}{|c|c|c|c|c|c|c|c|}
\hline Entry & Relay & Olefin & $\#$ & Product(s) & $\#$ & Yield $(\%)^{[b]}$ & $E / Z^{[c]}$ \\
\hline 1 & $3 a$ & & 6 & & 15 & 52 & n.d. \\
\hline 2 & $3 a$ & & 7 & & 16 & 92 & $70: 30$ \\
\hline 3 & $3 a$ & & 8 & & $\begin{array}{l}16 \\
(+17)^{[e]}\end{array}$ & $45^{[\mathrm{d}]}$ & n.d. \\
\hline 4 & $3 a$ & & 9 & & 17 & n.d..$^{[e]}$ & $\mathrm{n} / \mathrm{a}$ \\
\hline 6 & $3 a$ & & 11 & & 18 & 84 & 73:27 \\
\hline 7 & $3 a$ & & 12 & & 17 & n.d..$^{[e]}$ & $\mathrm{n} / \mathrm{a}$ \\
\hline 8 & $3 a$ & & 13 & & 19 & 64 & $73: 27$ \\
\hline 9 & 4 & & 11 & & $20 a$ & 24 & $\mathrm{n} / \mathrm{a}$ \\
\hline 10 & $5 a$ & & 11 & & 21 & 68 & $70: 30$ \\
\hline 11 & $5 a$ & & 13 & & 22 & 69 & $73: 27$ \\
\hline 12 & $5 b$ & & 11 & & 21 & 0 & $\mathrm{n} / \mathrm{a}$ \\
\hline 13 & $5 c$ & & 11 & & 21 & trace & n.d. \\
\hline 14 & $3 a$ & & 14 & & 23 & 66 & $66: 34$ \\
\hline 15 & $3 b$ & & 14 & & 23 & 60 & $67: 33$ \\
\hline
\end{tabular}

[a] 0.25 mmol scale, conditions: olefin (5 equiv.), Gll (1) (10 mol\%), neat, $50{ }^{\circ} \mathrm{C}, 1 \mathrm{~h}$; [b] Isolated yields after chromatography; [c] $E / Z$ ratio determined by ${ }^{1} \mathrm{H}$ NMR and assigned on the basis of characteristic ${ }^{13} \mathrm{C}$ NMR shielded methyl resonances for $E$-isomers (See SI); [d] Yield determined by ${ }^{1} \mathrm{H}$ NMR using mesitylene as an internal standard; [e] 'Truncated' compound 17 was not isolated due to its volatility but assigned on the basis of a characteristic ${ }^{1} \mathrm{H}$ resonance at $\delta 4.72$ ( $\mathrm{m}, 2 \mathrm{H}$ ) ppm; [f] $E / Z$ ratio determined by ${ }^{1} \mathrm{H}$ NMR and assigned on the basis of characteristic ${ }^{3} \mathrm{JH}_{\mathrm{H}-\mathrm{H}}$ coupling constants. 
The effect of temperature (entries 8-9), equivalents of citral (26) (entry 10) and catalyst loading (entry 11) were also explored, with lower yields obtained. The further addition of Cul[ ${ }^{[29]}$ (entry 12) was found to be beneficial, as was increasing the catalyst loading (20 mol\%, entry 13). Increasing quantities of added $\mathrm{Cul}$ and $\mathrm{AcOH}$ (entries 14-15) resulted in a higher yield, providing a final optimized yield of $88 \%$ (entry 15) for this challenging transformation. The use of the Hoveyda-Grubbs catalyst (2) (entry 16) under conditions that worked well (c.f., entry 7) for catalyst 1 surprisingly gave a complex and inseparable product mixture. To the best of our knowledge, this is the first protocol that allows for the ascent of the terpenes using naturally occurring terpene building blocks. We then turned our attention to demonstrating that the protocol is suitable for iteration (Scheme 2). Accordingly, aldehyde 27 was reduced ${ }^{[30]}$ and $O$-allylated to provide $\mathrm{C}_{15}$-relay metathesis substrate 29 . A second $\mathrm{ReXM}$ with citral (26) now produced $\mathrm{C}_{20}$-diterpene $\mathbf{3 0}$, ${ }^{[31]}$ which could be readily reduced to the $\mathrm{C}_{20}$-alcohol $\mathbf{3 1}$ as the first step of another iteration.

With the ability to synthesize enantiopure, $\Delta^{14,15}$ regioselectively functionalized geranylgeraniol $\mathbf{3 1}$, we now targeted macrocycle 35 (P = Et) - pertinent as a putative biogenetic precursor of the bromophycolide halogenated natural product family (Scheme 2$){ }^{\left[{ }^{32]}\right]}$ Thus alcohol $\mathbf{3 1}$ was activated as its bromide and coupled to an aryl iodide (see SI) to give diterpene benzoate 33. Alternatively, taking advantage of our previous observation that prenylbenzene was unreactive to the ReXM conditions (Table 1, entry 5), geranyl benzoate 32 was combined with relay sesquiterpenoid 29 to provide 33 in good yield. Subsequent ester hydrolysis and regioselective epoxide ring-opening with bromide gave bromohydrin $\mathbf{3 4}$. With the scene now set for macrocyclization, we anticipated that the inseparable $E / Z$ alkene isomers that had built up in the ReXM iteration sequence, ${ }^{[33]}$ would become chromatographically distinguishable upon conversion to conformationally constrained rings. Much to our delight, Shiina macrolactonization ${ }^{[34]}$ proceeded with excellent efficiency $(91 \%)$ and provided $(E, E, E)$-macrocycle $35(\mathrm{P}=\mathrm{Et})$ as

the major macrocyclic component which was readily separable from the other Z-olefin containing macrocycles (see $\mathrm{SI}$ ).

Table 2. Optimization of the ReXM reaction of allyl ethers $(S)-3 \mathbf{a}$ and $5 \mathbf{a}$ with citral (26). ${ }^{[a]}$

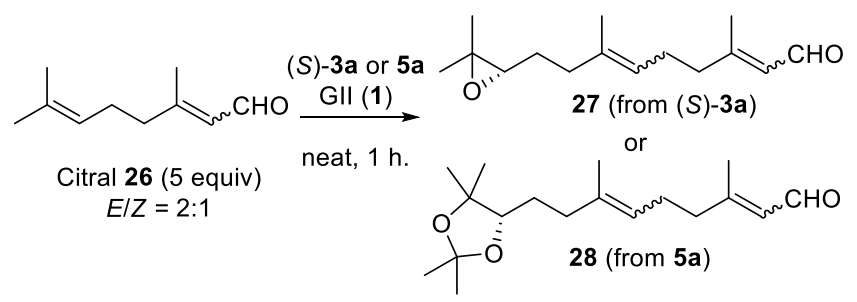

\begin{tabular}{|c|c|c|c|c|c|c|}
\hline Entry & 26 (equiv) & $\begin{array}{l}\text { Relay } \\
3 \mathbf{a} \text { or } 5 \mathbf{a}\end{array}$ & Ru cat. (mol\%) & $\mathrm{T}\left({ }^{\circ} \mathrm{C}\right)$ & $\begin{array}{l}\text { Additive(s) } \\
\text { (mol\%) }\end{array}$ & Yield(\% $)^{[b]}$ \\
\hline 1 & 5 & $3 a$ & $1(10)$ & 50 & - & $\mathrm{O}^{[\mathrm{c}]}$ \\
\hline 2 & 10 & $3 a$ & $1(10)$ & 50 & - & $0^{[c]}$ \\
\hline 3 & 5 & $3 a$ & $1(10)$ & $\mathrm{RT}$ & - & $0^{[c]}$ \\
\hline 4 & 5 & $3 a$ & $1(2)$ & 50 & - & $0^{[c]}$ \\
\hline 5 & 5 & $3 a$ & $1(10)$ & 50 & $p \mathrm{BQ}(20)$ & $0^{[c]}$ \\
\hline 6 & 5 & $3 a$ & $1(10)$ & 50 & $\mathrm{AcOH}(20)$ & 64 \\
\hline 7 & 5 & $5 a$ & $1(10)$ & 50 & $\mathrm{AcOH}(20)$ & 64 \\
\hline 8 & 5 & $3 a$ & $1(10)$ & 70 & $\mathrm{AcOH}(20)$ & 19 \\
\hline 9 & 5 & $3 a$ & $1(10)$ & $\mathrm{RT}$ & $\mathrm{AcOH}(20)$ & $0^{[c]}$ \\
\hline 10 & 10 & $3 a$ & $1(10)$ & 50 & $\mathrm{AcOH}(20)$ & 30 \\
\hline 11 & 5 & $3 a$ & $1(2)$ & 50 & $\mathrm{AcOH}(20)$ & 4 \\
\hline 12 & 5 & $3 a$ & $1(10)$ & 50 & $\begin{array}{c}\mathrm{AcOH}(20), \mathrm{Cul} \\
(15)\end{array}$ & 68 \\
\hline 13 & 5 & $3 a$ & $1(20)$ & 50 & $\mathrm{AcOH}(20)$ & 80 \\
\hline 14 & 5 & $3 a$ & $1(20)$ & 50 & $\begin{array}{c}\mathrm{AcOH}(20), \mathrm{Cul} \\
(30)\end{array}$ & 84 \\
\hline 15 & 5 & $3 a$ & $1(20)$ & 50 & $\begin{array}{c}\mathrm{AcOH}(40), \mathrm{Cul} \\
(30)\end{array}$ & 88 \\
\hline 16 & 5 & $5 a$ & $2(10)$ & 50 & $\mathrm{AcOH}(20)$ & $0^{[c]}$ \\
\hline
\end{tabular}

[a] Reactions conducted on a $0.25 \mathrm{mmol}$ scale; [b] Isolated yields after chromatography, E/Z ratio determined as ca. $3: 1$ at the newly formed olefin ( $\left.\Delta^{6,7}\right)$ and unchanged as ca. 2:1 at the $\alpha, \beta$-unsaturated aldehyde by ${ }^{1} \mathrm{H}$ NMR and assigned on the basis of characteristic ${ }^{13} \mathrm{C}$ NMR shielded methyl resonances for $E$ isomers (See SI); [c] Purification not attempted due to complex mixtures of products. 
(S)-3a

$$
1(20 \mathrm{~mol} \%)
$$

26 (5 equiv)

$\mathrm{AcOH}(40 \mathrm{~mol} \%)$

Cul $(30 \mathrm{~mol} \%)$

$50{ }^{\circ} \mathrm{C}, 1 \mathrm{~h}$.

$88 \%$

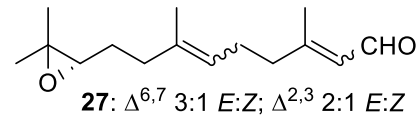

27: $\Delta^{6,7} 3: 1 E: Z ; \Delta^{2,3} 2: 1 E: Z$

1. DOWEX-50W, $\mathrm{NaBH}_{4}$,

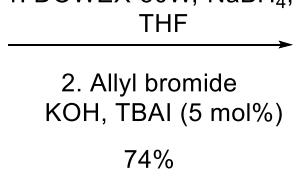

$74 \%$

(over 2 steps)

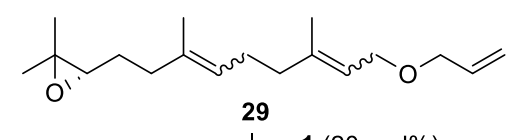

29

$1(20 \mathrm{~mol} \%)$

$\mathrm{AcOH}(40 \mathrm{~mol} \%)$

Cul $(30 \mathrm{~mol} \%)$

$50{ }^{\circ} \mathrm{C}, 30 \mathrm{~min}$.

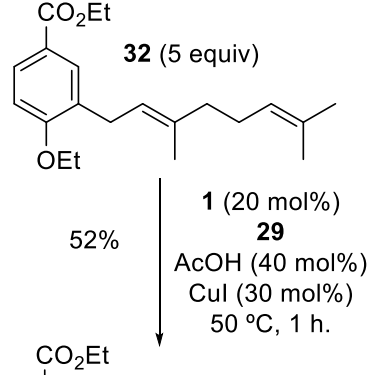

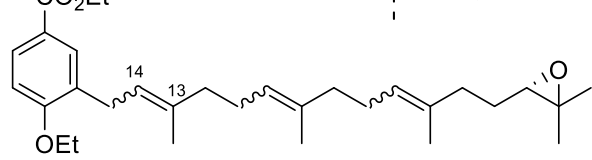

$33\left(\Delta^{13,14} 100 \% E\right.$ from 32$)$

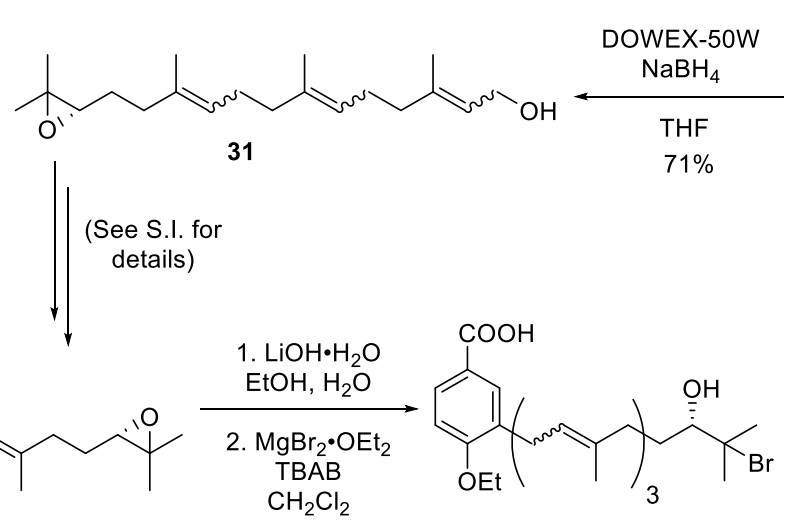

34 (79\% over two steps)
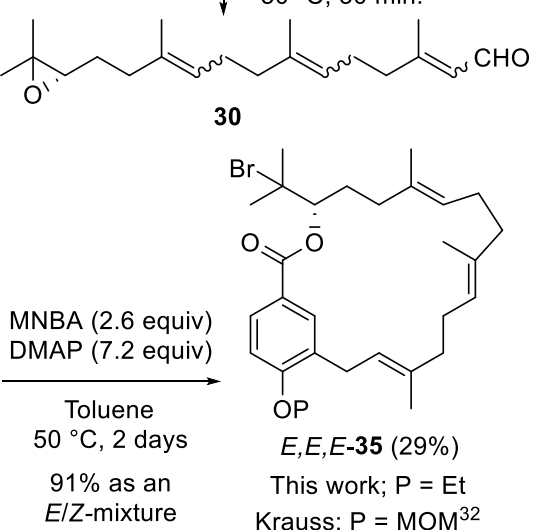

Scheme 2. ReXM for the ascent of a monoterpene to a sesquiterpene to a diterpene and subsequent synthesis of a diterpene-benzoate macrolide pertinent to the bromophycolide family of natural products. TBAI = tetra- $n$-butylammonium iodide; TBAB = tetra- $n$-butylammonium bromide; MNBA = 2-methyl-6-nitrobenzoic anhydride; DMAP = 4-dimethylaminopyridine. 


\section{Conclusion}

In conclusion, we have designed and demonstrated an unprecedented olefin-olefin combination to form unfunctionalized trisubstituted olefins by cross metathesis. This novel relay cross metathesis ("ReXM") reaction, utilizes two different trisubstituted olefins, one of which is a trisubstituted allylic alcohol furnished with a relay, while the non-relay partner is an unfunctionalized trisubstituted olefin. This methodology allows for the relay to be directly incorporated on inexpensive terpenoid building blocks such as geraniol. Moreover, by using the readily available, inexpensive and naturally occurring terpenoid citral as the non-relay partner, this methodology allows the further unprecedented ascent of terpenoids (from a monoterpene, to a sesquiterpene, to a diterpene) via an iterative ReXM-reduction-relay installation sequence. We have used the method to construct an enantiomerically and geometrically pure diterpene benzoate macrolide of relevance to bioactive substances from marine organisms. The method reported should allow for the synthesis of myriad bespoke terpenes and facilitate the ability to valorize terpene biomass. ${ }^{[35]}$

\section{Acknowledgements}

We thank CSIRO and Imperial College London for a studentship (to K.A.B.), the China Scholarship Council for a scholarship (to Z.S.) and the EPSRC (Grant No. EP/P030742/1 to D.C.B.) for financial support.

[1] R. Firn, Nature's Chemicals: The Natural Products that Shaped Our World; Oxford University Press: Oxford, 2010.

[2] E. Breitmaier, Terpenes: Flavors, Fragrances, Pharmaca, Pheromones; Wily-VCH: Weinheim, 2006.

[3] For a pictorial 'Top pharmaceuticals' see: http://njardarson.lab.arizona.edu/content/top-pharmaceuticals-poster (11th June 2019).

[4] D. H. R. Barton, O. Meth-Cohn, K. Nakanishi, Isoprenoids Including Cartenoids and Steroids, in Comprehensive Natural Product Chemistry; Vol. 2. (Eds.: Cane, D. E.), Pergamon: Elmsford, NY, 1999.

[5] H. M. Miziorko, Arc. Biochem. Biophys., 2011, 505, 131-143.

[6] Q. Zhang, L. Huang, C. Zhang, P. Xie, Y. Zhang, S. Ding, F. Xu, Fitoterapia, 2015, 106, 184-193.

[7] J. F. Pennock, F. W. Hemming, R. A. Morton, Nature, 1960, 186, 470-472.

[8] V. Cantagrel, D. J. Lefeber, J. Inherit. Metab. Dis., 2011, 34, 859-867.

[9] (a) For a solid-phase synthesis of solanesol see: X. Yu, S. Wang, F. Chen, J. Comb. Chem., 2008, 10, 605-610. (b) For the use of organometallic methodology using specialized iodoalkene fragments see: E.-I. Negishi, S.-Y. Liou, S. Hui, Org. Lett., 2002, 4, $261-264$.

[10] O. M. Ogba, N. C. Warner, D. J. O'Leary, R. H. Grubbs, Chem. Soc. Rev., 2018, 47, 4510-4544.

[11] T. Ritter, A. Hejl, A. G. Wenzel, T. W. Funk, R. H. Grubbs, Organometallics, 2006, 25, 5740-5745.

[12] (a) For $E$ - and Z-trisubstituted olefins by cross metathesis using molybdenum alkylidene pre-catalysts see: T. T. Nguyen, M. J. Koh, T. J. Mann, R. R. Schrock, A. H. Hoveyda, Nature, 2017, 552, 347-355. (b) For the formation of E- and Z-trisubstituted allylic alcohols using a ruthenium catechothiolate complex see: Xu, C.; Liu, Z.; Torker, S.; Shen, X.; Xu, D.; Hoveyda, A. H. J. Am. Chem. Soc., 2017, 139, 15640-15643. (c) For $\alpha, \beta$-unsaturated carbonyl trisubstituted olefins by ruthenium benzylidene catalysed cross-metathesis of citronellal, citronellol and citral with methyl methacrylate see: H. Bilel, N. Hamdi, F. Zagrouba, C. Fishmeister, C. Bruneau, Green Chem., 2011, 13, 1448-1452. (d) For a trisubstituted olefin by ruthenium benzylidene catalysed cross-metathesis of citronellal with methacrolein see: J. Xu, E. J. E. Caro-Diaz, L. Trzoss, E. A. Theodorakis, J. Am. Chem. Soc., 2012, 134, 5072-5075. (e) For a recent review on metathesis of terpenes see: C. Bruneau, C. Fischmeister, D. Mandelli, W. A. Carvalho, E. N. dos Santos, P. H. Dixneuf, L. Sarmento Fernandes, Catal. Sci. Technol., 2018, 8, 3989-4004. See also ref. 21.

[13] A. K. Chatterjee, R. H. Grubbs, Org. Lett., 1999, 1, 1751-1753.

[14] Grubbs subsequently showed that 2-methyl-2-butene could also be employed: A. K. Chatterjee, D. P. Sanders, R. H. Grubbs, Org. Lett., 2002, 4, 1939-1942.

[15] Z. J. Wang, W. R. Jackson, A. J. Robinson, Org. Lett., 2013, 15, 3006-3009.

[16] For the original relay strategy as applied to relay ring closing metathesis see: T. R. Hoye, C. S. Jeffrey, M. A. Tennakoon, J. Wang, H. Zhao, J. Am. Chem. Soc., 2004, 126, 10210-10211.

[17] For a recent kinetic benchmarking of the prenyl group in RCM see: K. A. Bahou, D. C. Braddock, A. G. Meyer, G. P. Savage, Org. Lett., 2017, 19, 5332-5335.

[18] Diver and co-workers have reported the use of an allyl relay grouping for prenyl alcohol to surmount the unreactive nature of geminal olefins in ene-yne cross metathesis: J. R. Clark, J. M. French, E. Jecs, S. T. Diver, Org. Lett., 2012, 14, 4178-4181.

[19] There is only further limited literature precedent for relay cross metathesis all of which provide conjugated alkene products: (a) R. K. Malla, J. N. Ridenour, C. D. Spilling, Beilstein J. Org. Chem., 2014, 10, 1933-1941. For the construction of conjugated Z-enynes: (b) E. C. Hansen, D. Lee, Org. Lett., 2004, 6, 2035-2038. (c) H. Kim, H. Lee, D. Lee, S. Kim, D. Kim, J. Am. Chem. Soc., 2007, 129, 22692274. (d) A. Senapati, S. Das, C. V. Ramana, J. Org. Chem., 2018, 83, 12863-12868.

[20] H. S. Rao, S. P. Senthilkumar, J. Chem. Sci., 2001, 113, 191-196.

[21] A. K. Chatterjee, T.-L. Choi, D. P. Sanders, R. H. Grubbs, J. Am. Chem. Soc., 2003, 125, 11360-11370.

[22] For the degradation of precatalyst 1 with primary alcohols see: M. B. Dinger, J. C. Mol, Eur. J. Inorg. Chem., 2003, $2827-2833$.

[23] The $E: Z$ ratio of trisubstituted alkenes was determined by ${ }^{1} \mathrm{H}$ NMR integration of selected signals. The $E$ isomers were identified on the basis of their characteristically shielded methyl groups of the trisubstituted olefin in the ${ }^{13} \mathrm{C}$ NMR spectrum. See e.g., for $E$ - and Z-19: Y. Watanabe, S. Laschat, M. Budde, O. Affolter, Y. Shimadac, V. B. Urlachera, Tetrahedron, 2007, 9413-9422.

[24] 2,5-Dihydrofuran 24 (b.p. 66-67 ${ }^{\circ} \mathrm{C}$ ) can be observed by ${ }^{1} \mathrm{H}$ NMR (if the reaction is not purged of volatiles) after direct dissolution of the crude reaction mixtures in $\mathrm{CDCl}_{3}\left(\delta_{\mathrm{H}} 5.87(\mathrm{~s}, 2 \mathrm{H}), 4.62(\mathrm{~s}, 4 \mathrm{H})\right)$. Isobutylene 25 as a more volatile component $\left(\mathrm{b} . \mathrm{p} .-7^{\circ} \mathrm{C}\right)$ is not observed as expected.

[25] The use of citral (26) as an $E / Z$ enal mixture should be unimportant in the subsequent ascent sequence, since the same ruthenium alkylidene(s) (of the type B, Scheme 1) should be formed after initial relay metathesis from either of the original $\Delta^{2,3}$ geometries of e.g. farnesol allyl ether 29. The experiments conducted with nerol vs geraniol derived $O$-allyl epoxides (Table 1, entries 14-15) are consistent with this expectation.

[26] Citral has recently been reported as a cross metathesis partner with terminal alkenes: R. R. Sapkota, J. M. Jarvis, T. M. Schaub, M. R. Talipov, J. B. Arterburn, Chem. Open, 2019, 8, 201-205. 
[27] 2,3-Dihydrofuran was identified by the presence of a characteristic ${ }^{1} \mathrm{H}$ NMR resonance in $\mathrm{CDCl}_{3}$ of crude reaction mixtures $\left[\delta_{\mathrm{H}} 4.16(\mathrm{t}\right.$, $J=9.6 \mathrm{~Hz}, 2 \mathrm{H}) \mathrm{ppm}]$.

[28] S. H. Hong, D. P. Sanders, C. W. Lee, R. H. Grubbs, J. Am. Chem. Soc., 2005, 127, 17160-17161.

[29] K. Voigtritter, S. Ghorai, B. H. Lipshutz, J. Org. Chem., 2011, 76, 4697-4702.

[30] B. Zeynizadeh, F. Shirini, Bull. Korean Chem. Soc., 2003, 24, 295-298.

[31] After the second iteration it was no longer possible to determine the three $E: Z$ ratios at $\Delta^{2,3}, \Delta^{6,7}$ and $\Delta^{10,11}$ in compound $\mathbf{3 0}$ by NMR methods.

[32] Macrocycle 35, as a MOM-protected phenol, has previously been reported: H. Lin, S. S. Pochapsky, I. J. Krauss, Org. Lett., 2011, 13, $1222-1225$.

[33] On the basis of an approximate 3:1 $E: Z$ alkene ratio for each of the two ReXM iterations and an approximate $2: 1 \quad E: Z$ alkene ratio for olefin derived from the $\alpha, \beta$-unsaturated portion of citral, we estimate $(E, E, E)-34$ to be the major component of the mixture at $37 \%$.

[34] I. Shiina, M. Kubota, H. Oshiumi, M. Hashizume, J. Org. Chem., 2004, 69, 1822-1830

[35] ${ }^{1} \mathrm{H}$ NMR, ${ }^{13} \mathrm{C}$ NMR, IR and MS data are available via a data repository as: K. A. Bahou, D. C. Braddock, Z. Shi Imperial College HPC Data Repository, 2019. DOI: 10.14469/hpc/5737 (accessed 18/06/2019). 